\title{
Diffraction Contrast Imaging Using Virtual Apertures
}

\author{
Christoph Gammer ${ }^{1,2,3 *}$, V. Burak Ozdol1, Christian H. Liebscher ${ }^{1,2,4}$, Andrew M. Minor ${ }^{1,2}$ \\ ${ }^{1}$ National Center for Electron Microscopy, Molecular Foundry, Lawrence Berkeley National \\ Laboratory \\ ${ }^{2}$ Department of Materials Science and Engineering, University of California, Berkeley \\ ${ }^{3}$ Physics of Nanostructured Materials, Faculty of Physics, University of Vienna, Austria. \\ ${ }^{4}$ present address: Interdisciplinary Center for Analytics on the Nanoscale (ICAN), Center for \\ Nanointegration Duisburg-Essen (CENIDE), Carl-Benz-Str. 199, University Duisburg-Essen, \\ Germany \\ *Corresponding author: cgammer@lbl.gov, christoph.gammer@univie.ac.at
}

\begin{abstract}
Two methods on how to obtain the full diffraction information from a sample region and the associated reconstruction of images or diffraction patterns using virtual apertures are demonstrated. In a STEM-based approach, diffraction patterns are recorded for each beam position using a small probe convergence angle. Similarly, a tilt series of TEM dark-field images is acquired. The resulting datasets allow the reconstruction of either electron diffraction patterns, bright-, darkor annular dark-field images using virtual apertures. The experimental procedures of both methods are presented in the paper and are applied to a precipitation strengthened and creep deformed ferritic alloy with a complex microstructure. The reconstructed virtual images are compared with conventional TEM images. The major advantage is that arbitrarily shaped virtual apertures generated with image processing software can be designed without facing any physical limitations. In addition, any virtual detector that is specifically designed according to the underlying crystal structure can be created to optimize image contrast.
\end{abstract}

\section{Keywords}

diffraction imaging, nanodiffraction, dark field scanning, precipitates, large data, virtual microscopy

\section{Introduction}

Transmission electron microscopy (TEM) is an important tool for the analysis of a wide range of materials. In the field of materials science conventional TEM (CTEM) is routinely used to study structural properties of materials with nanometer resolution by combining electron diffraction and diffraction contrast imaging techniques [1-3]. Electron diffraction patterns of crystalline materials contain information about the crystal symmetry, lattice parameters and orientation relationships of multi-phase materials. Traditionally, this information can be acquired from specifically selected regions using a selected area aperture where the resolution is limited by the effective size of the aperture, which itself is ultimately limited by lens aberrations. Once the crystallographic characteristics are determined, diffraction contrast imaging can be used to form bright- and dark- 
field images by selecting specific reflections with the objective aperture, which is typically done in a so-called "two-beam" condition. This technique allows for visualization of the grain structure, different phases and lattice defects, like planar faults or dislocations, from selected regions with high resolution.

Alternatively, scanning transmission electron microscopy (STEM) uses a converged electron probe that is scanned over the sample rather than apertures to form the image. Annular detectors are often used for imaging and by varying the camera length, the collection angle of the detector can be changed. The segmentation of STEM detectors allows for the simultaneous imaging of bright- and dark-field signals, but also the selection of an angular range of scattered electrons [4,5]. High angle annular dark-field (HAADF) STEM provides a way of imaging Z-contrast by collecting incoherently scattered electrons with scattering angles $>50$ mrad being dominated by Rutherford scattering, which is frequently exploited for atomic resolution imaging [6]. For annular dark-field (ADF) STEM, Bragg scattered electrons are used for image formation and the image contrast is determined by diffraction contrast. This method can be used to image defects in crystalline materials with reduced dynamical scattering contrast compared to CTEM and is well suited for imaging dislocations [7-9]. STEM imaging also can be used to form diffraction patterns with a sub-nm sized probe, which allows one to analyze nanosized volumes [10,11]. Rodenburg et al. [12] and Cowley et al. [13] combined STEM imaging and Nanodiffraction to increase the resolution of conventional STEM imaging by collecting diffraction patterns for equally spaced probe positions. This led to an increase in resolution by a factor of two or more over regular STEM imaging.

The characterization of phase and defect arrangements in a material typically requires tilting the sample to varying operating reflections. This means that a dataset of several static diffraction patterns and associated TEM images are acquired and afterwards evaluated. Once the dataset is taken, it can be difficult to extract additional information without finding the same region of interest in a separate experimental setup. In the case of CTEM the image is formed on the detector for one specific aperture position. In contrast, for ADF-STEM the image is formed by recording one value extracted from the annular detector for each beam position and the diffraction information in the detector hole is typically not recorded.

The present paper presents a method to acquire diffraction patterns for each beam position in STEM with a small convergence angle that can overcome the limitations of CTEM and ADF-STEM, creating a rich diffraction-based dataset that can be analyzed and presented in multiple ways after the experiment [14]. In order to demonstrate the usefulness of this technique, a precipitation strengthened and creep deformed alloy [15] with a complex microstructure was used to test and verify the method. Both electron diffraction patterns and virtual images can be reconstructed for a given sample orientation after the experiment without facing the limitation in the size or shape of the physical apertures. The advantage here is that arbitrarily-shaped virtual apertures, generated with the image processing software, can be used to reconstruct either electron diffraction patterns, bright- or dark-field images. In addition, we compare this method to a dataset that is acquired in TEM mode in a similar manner to that described in [16-18], where conventional dark-field images are recorded with varying beam tilt angle. Similarly, virtual electron diffraction patterns or images can be reconstructed from the dataset. 


\section{Experimental procedures}

The ferritic alloy Fe-10Cr-10Ni-5.5Al-3.4Mo-0.25Zr-0.005B (wt.\%) was drop-cast by Sophisticated Alloys (Butler, PA). The cast billet was homogenized at $1200^{\circ} \mathrm{C}$ for $0.5 \mathrm{~h}$ in evacuated quartz tubes, air-cooled and subsequently aged at $700{ }^{\circ} \mathrm{C}$ for $100 \mathrm{~h}$ followed by air-cooling. A dogbone specimen was cut from the ingot with a gauge length of $35 \mathrm{~mm}$ and cross-section of $6.6 \mathrm{~mm}$. The sample was creep deformed under tensile load in air with a stress level of $107 \mathrm{MPa}$ at $700^{\circ} \mathrm{C}$. The test was interrupted after $2.3 \%$ strain and the sample was air cooled under load to preserve the high temperature dislocation structure. More details about the sample are given in the paper by Vo et al. [15]. TEM samples with a diameter of $3 \mathrm{~mm}$ were cut from the gauge length by a rotary disc cutter. The samples were thinned to electron transparency by electropolishing using an electrolyte of $20 \%$ percholoric acid in methanol at $-30{ }^{\circ} \mathrm{C}$.

Zero-loss filtered TEM imaging was performed in a Zeiss Libra200MC microscope at an acceleration voltage of $200 \mathrm{kV}$ equipped with an in-column omega energy filter. A tilt series of dark-field images were recorded using the QED (Quantitative Electron Diffraction) plug-in for Digital Micrograph that allows for automatic control of the electron beam [16,17]. The software controls the beam tilt for each image acquired in the series and compensates for aberrations of the illumination system such as tilt-induced illumination shift up to large beam tilt angles. A series of 1961 dark-field images were acquired with a tilt angle up to $\pm 30 \mathrm{mrad}$ (corresponding to $\pm 12 \mathrm{~nm}^{-1}$ ) and a step size of 1.2 mrad using a $10 \mu \mathrm{m}$ aperture (corresponding to a diameter of $1.30 \mathrm{~nm}^{-1}$ in reciprocal space). The images (1024x1024 pixel, 1.5x1.5 $\mu \mathrm{m})$ were recorded using a Gatan Orius CCD camera with an exposure time of $5 \mathrm{~s}$ and $1 \mathrm{~s}$ for the dark- and bright-field images, respectively. Sample drift was compensated for after acquiring the image series where the drift of successive images was compared using cross-correlation. To enhance the precision of the drift compensation, pre-filtering of the images using a bandpass filter was carried out and a clear feature was selected for crosscorrelation. An on-line drift correction during the acquisition, as available in QED, was not employed. The datacube was evaluated using custom written scripts in DigitalMicrograph allowing for arbitrarily-shaped virtual apertures.

\section{STEM imaging was performed in an FEI Titan 80-300 microscope equipped with a FEG operated at $300 \mathrm{kV}$. Its three-condenser system allows for control of the semi-convergence angle to form sub-nm sized electron probes even at small convergence angles. In the present work a semi-convergence angle of $1.2 \mathrm{mrad}$ was chosen in order to minimize the probe size yet still have clearly separated diffracted discs. For each individual pixel in an ADF-STEM image (256x256 pixel) diffraction patterns were recorded simultaneously at a camera length of $380 \mathrm{~mm}$ setting the inner angle of the ADF}



detector at $17 \mathrm{mrad}$ (corresponding to $8.5 \mathrm{~nm}^{-1}$ ). The diffraction patterns (1920x1796 pixel) were recorded using Gatan K2-IS direct electron detector operating at a frame rate of $400 \mathrm{f} / \mathrm{s}$. The high speed of the camera allowed for recording of the datacube consisting of 65536 single diffraction patterns in only 164 seconds. To evaluate the large datacube, custom DigitalMicrograph scripts were written. Most evaluations were carried out on a dataset that was rebinned by 8. The sample was oriented in [100] zone-axis for both STEM and TEM mapping. Regular ADF- and HAADF-STEM images were recorded using a semi-convergence angle of $17 \mathrm{mrad}$ and inner semi-collection angles of 17 and $61 \mathrm{mrad}$, respectively.Imaging methods

\subsection{TEM diffraction mapping}

In TEM diffraction mapping a series of dark-field images is recorded for a variable number of beam tilts. The beam tilt pattern is set up to be a circular region with a specified maximum tilt angle, filled with a rectangular grid with a specified tilt spacing. The resulting data cube is composed of a set of aberration-compensated dark-field images associated to a specific beam tilt angle. The dark-field images are aligned by cross correlation to compensate for sample drift. Two sets of virtual apertures can be used to reconstruct images as illustrated in Figure 1a. A virtual selected area aperture can be placed in any of the recorded dark-field images and the corresponding selected area diffraction pattern is generated after the experiment by averaging the intensity of this region for each beam tilt. Specifically, each pixel value in the virtual diffraction pattern corresponds to the mean intensity of the selected region for the corresponding beam tilt. A virtual objective aperture can then be placed in any region of the resulting diffraction pattern and the corresponding darkfield image is obtained by summing the images with the respective beam tilt angles as selected by the aperture.

\subsection{STEM diffraction mapping}

In STEM diffraction mapping a sample region is scanned by a converged electron probe and for each probe position an electron diffraction pattern is recorded along with the regular ADF-signal as demonstrated in Figure 1b. Thus, each pixel of the resulting STEM map is a full diffraction pattern for the corresponding beam position. By calculating the mean intensity for a virtual aperture at each probe position the virtual dark-field image can be reconstructed. Once the dark-field image is obtained, a virtual selected area aperture can be placed in any region of the image and the respective diffraction pattern can be calculated by summing all diffraction patterns from that region (Figure 1b). 
The two methods are similar since virtual diffraction patterns or images are obtained from a series of images, in the case of TEM a tilt series of dark-field images and in the case of STEM a scanned series of diffraction patterns. In fact, the mechanism behind the two methods is comparable to the reciprocity of TEM and STEM. In one technique the beam is tilted before the specimen plane and the resulting image is recorded, and with the other technique the sample is scanned in the specimen plane and the diffracted beams are recorded. It should be pointed out that in STEM diffraction mapping all diffracted beams correspond to the same range of incident beam directions while in TEM diffraction mapping the beam is tilted to obtain different dark-field images.

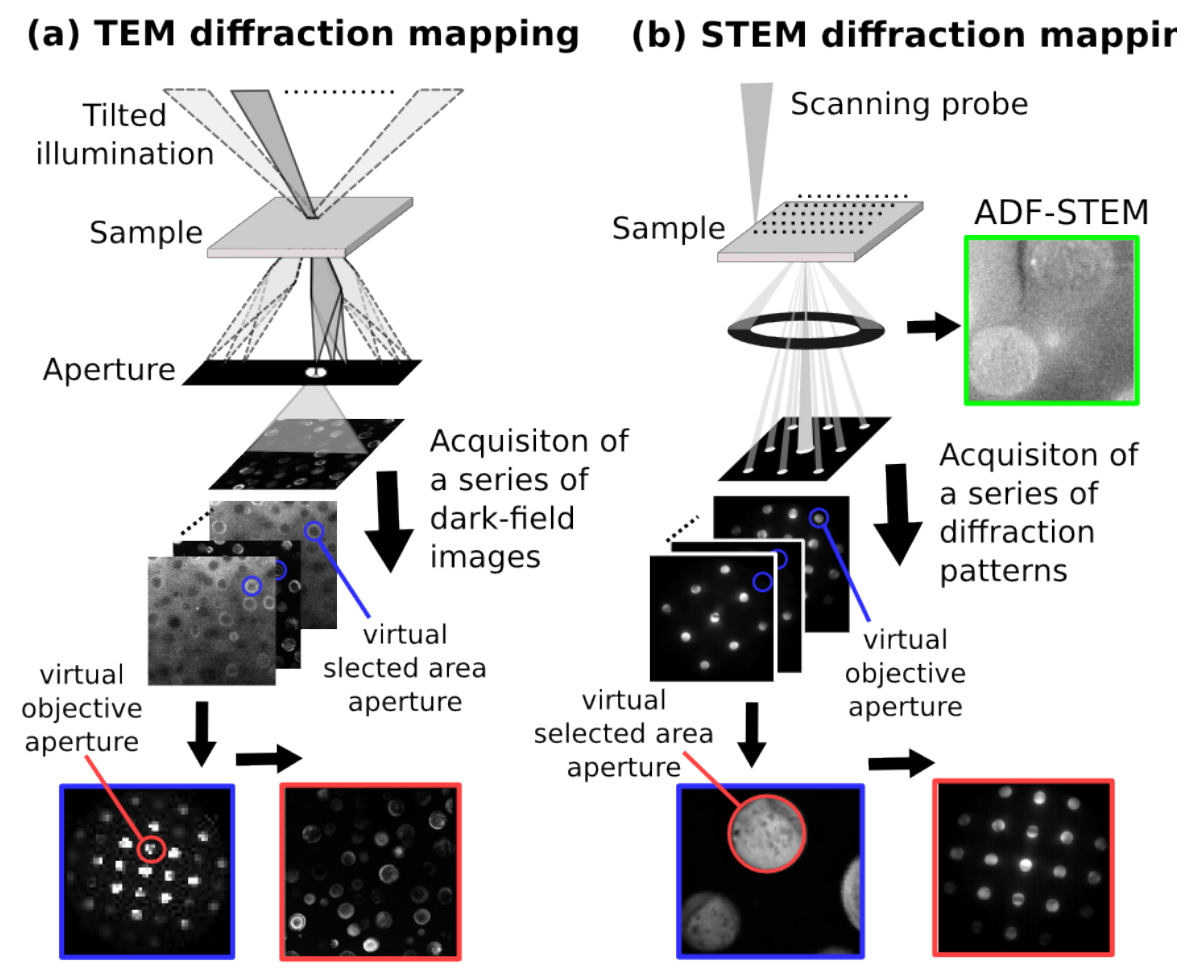

Figure 1: Experimental Setup: (a) TEM diffraction mapping: Rather than acquiring a single image, a series of dark-field images is recorded by tilting the illumination. Diffraction patterns can be reconstructed by setting a virtual selected area aperture (blue circle) and calculating the mean intensity in the aperture for each dark-field image. Selecting a certain region in the diffraction pattern and summing those dark-field images sets a virtual objective aperture. (b) STEM diffraction mapping: In conventional STEM, a probe scans over the sample and an ADF image (green) is acquired using an annular dark-field detector. In the current setup, in addition a diffraction pattern is recorded for every probe position. By calculating for every probe position the mean intensity in a virtual objective aperture (blue circle) a virtual dark-field image can be reconstructed. Selecting a certain area in the image (red circle) and summing all corresponding diffraction patterns, allows setting a virtual selected area aperture. 


\section{Results}

\subsection{Microstructure}

Before we focus on the results of the two methods employing virtual apertures, HAADF- and ADFSTEM images of a similar region of the alloy are presented as a reference to compare with the reconstructed images presented in the following sections. Figure 2 illustrates a comparison of a HAADF-STEM image (collection angle range 64-400 mrad) taken with a large camera length and therefore large collection angle (Figure 2a) and an ADF-STEM signal (collection angle range 17-106 $\mathrm{mrad}$ ) from the same sample region (Figure $2 \mathrm{~b}$ ). The microstructure consists of primary B2-NiAl precipitates, with associated $\{010\}$ superlattice reflections (Figure $2 c$ ) which are coherently embedded in the bcc-Fe matrix. In Figure $2 b$ the dislocation-particle interaction is clearly resolved with a small camera length where the image contrast is dominated by diffraction contrast. (Detailed description of the microstructure and creep properties can be found in a previous paper [15]). Although almost no residual dislocation strain contrast is observed in the HAADF image of Figure 2a, smaller B2-NiAl precipitates appear bright with respect to the bcc-Fe matrix. This observation suggests that contrast from elastic strain at the matrix-precipitate interface is superimposed on the Z-contrast as observed by Perovic et al. [9]. Pure Z-contrast should lead to a lower intensity of the smaller B2-precipitates with respect to the matrix due to their smaller mean Z. Interestingly, white contrast lines are observed around several smaller precipitates in the ADFsignal of Figure $2 \mathrm{~b}$ where image contrast is dominated by strain contrast supporting that strain fields of coherent particles with elastic misfit are observable in ADF-STEM imaging.

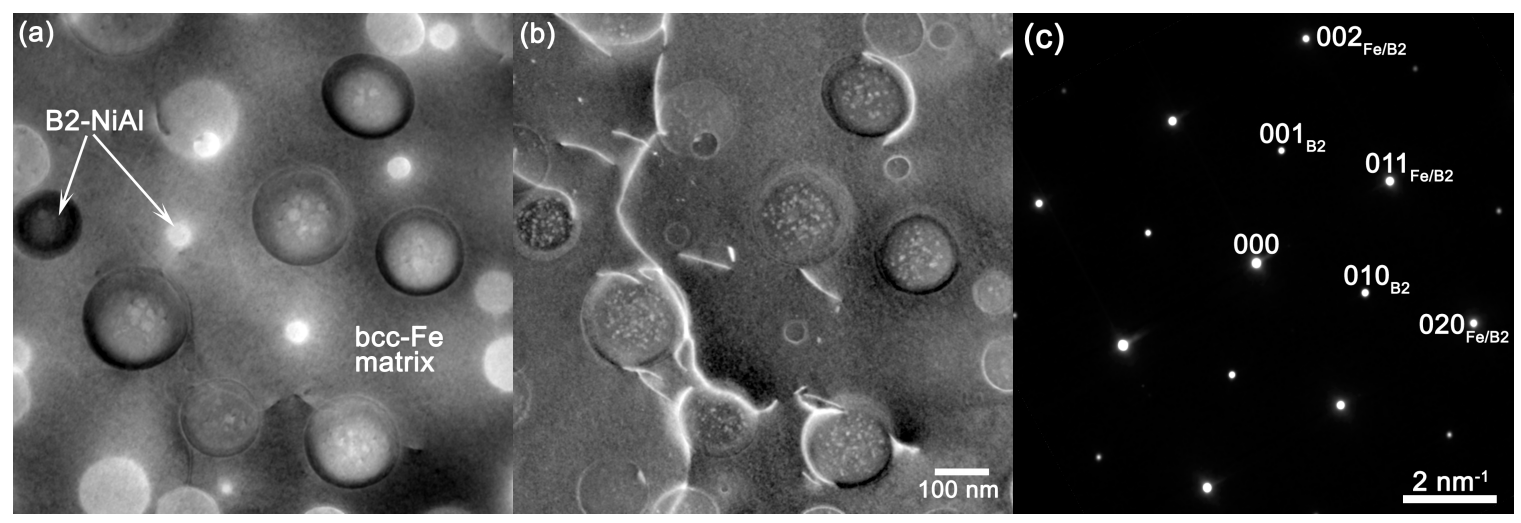

Figure 2: a) HAADF- and b) ADF-STEM images in [100] zone axis orientation of a representative region of the precipitation strengthened ferritic alloy after creep deformation. As expected the HAADFimage is dominated by Z-contrast revealing the microstructure of the alloy and the ADF-signal shows strong diffraction contrast and thus the location of dislocations in the same sample region. c) [100] zone axis selected area diffraction pattern indicating the $\{010\}_{B 2}$ superlattice and the $\{020\}$ fundamental reflections. 


\subsection{TEM Diffraction Mapping}

In the first part of the paper we analyze the sample in TEM mode by acquiring a series of dark-field images at a given sample area. Figure 3 shows a representative bright-field image from the tilt series along with reconstructed diffraction patterns from the precipitation-strengthened ferritic alloy. The bright-field image shown in Figure 3a reveals the presence of the spherical primary precipitates with an average diameter of $124 \mathrm{~nm}$, a small triangular shaped precipitate (approximately $30 \mathrm{~nm}$ ) and residual dislocation contrast. Figure $3 \mathrm{~b}$ shows the diffraction pattern corresponding to the matrix structure that was calculated by selecting a square region (blue square in (a)). The diffraction pattern is in [100] zone axis orientation and confirms that the structure of the matrix is bcc. Figure 3c shows the diffraction pattern obtained for the circular region corresponding to a precipitate (red circle in (a)). Additional spots are visible when compared to Figure $3 \mathrm{~b}$ revealing that the precipitates have a B2-ordered superlattice structure. Figure $3 \mathrm{~d}$ is the diffraction pattern corresponding to the small triangular precipitate (green region in (a)). The diffraction pattern exhibits superlattice reflection corresponding to the structure of a $\mathrm{Fe}_{23} \mathrm{Zr}_{6}$-type precipitate as described in $[15,19]$.

The results show how diffraction patterns can be reconstructed from the entire datacube for arbitrarily shaped areas selected in the image after the experiment. The fact that in the current approach the region is selected digitally has the significant advantage that there is no limitation on the size or shape of the aperture. Furthermore, diffraction patterns can be generated after the experiment using the information in the acquired datacube. 


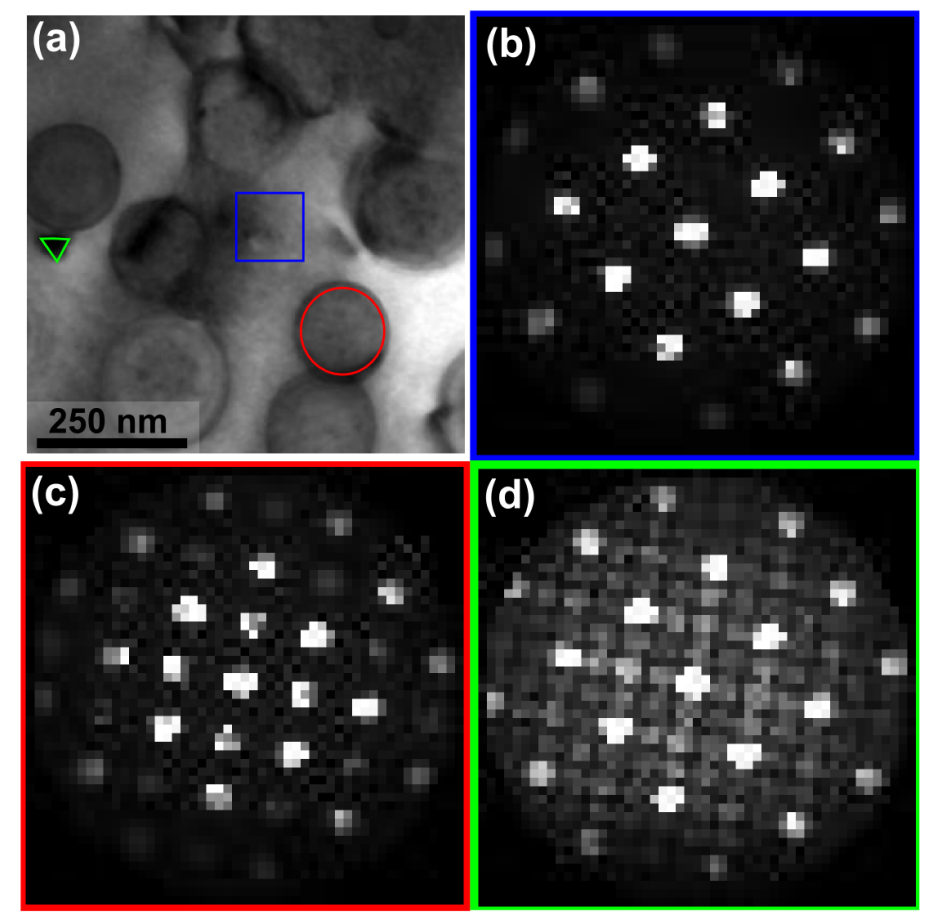

Figure 3: Virtual SA aperture: A series of dark-field images was recorded for a given sample position allowing to generate diffraction patterns from any region in the image. (a) Bright-field image of a precipitation strengthened Fe-Al-Ni-Cr alloy. (b) Matrix diffraction pattern (DP) calculated from the region indicated in blue in (a) showing a bcc structure in 100 orientation. (c) DP calculated from the precipitate selected with the red circle in (a). The DP shows a B2 superlattice structure. (d) DP resulting from a small triangular precipitate (selected with a green triangle in (a)). The DP corresponds to the structure of $\mathrm{Fe}_{23} \mathrm{Zr}_{6}$.

The datacube obtained with TEM diffraction mapping contains all dark-field images from the tilt series and it is therefore possible to display individual dark-field images after the dataset was recorded. In addition to that it is possible to sum multiple dark-field images with specific beam incidence conditions that can be chosen using a virtual objective aperture. Figure 4 shows a comparison of an individual dark-field image of the ferritic alloy with images formed by summing dark-field images from multiple diffraction conditions. The area shown in Figure $3 \mathrm{a}$ is a cutout of the current image. Figure $4 \mathrm{a}$ is a dark-field image formed using a $\{100\}$ reflection corresponding to the B2-phase of the spherical precipitates. In Figure 4b, all four $\{100\}$-type reflections were selected and the corresponding dark-field images were summed. Dynamic effects are reduced and the precipitates are more clearly visible with a homogeneous contrast, while in (a) some precipitates are only partially or not visible at all, because of extinction effects. The results show how virtual objective apertures can be used to sum multiple dark-field images with specific beam incidence conditions. In this way it is possible to overcome the limitations of static CTEM where only one specific diffracted beam can be selected for imaging. Instead, multiple reflections can be chosen or more complex apertures can be used, e.g. a conical dark-field image can be formed using an annular 
virtual detector. Figure 4c shows a conical dark-field image formed by placing the annular virtual detector at a semi-convergence angle of $19 \mathrm{mrad}$. The resulting image clearly reveals the dislocation structures and the strain contrasts around the precipitates, similarly to the ADF-STEM image shown in Figure 2b.
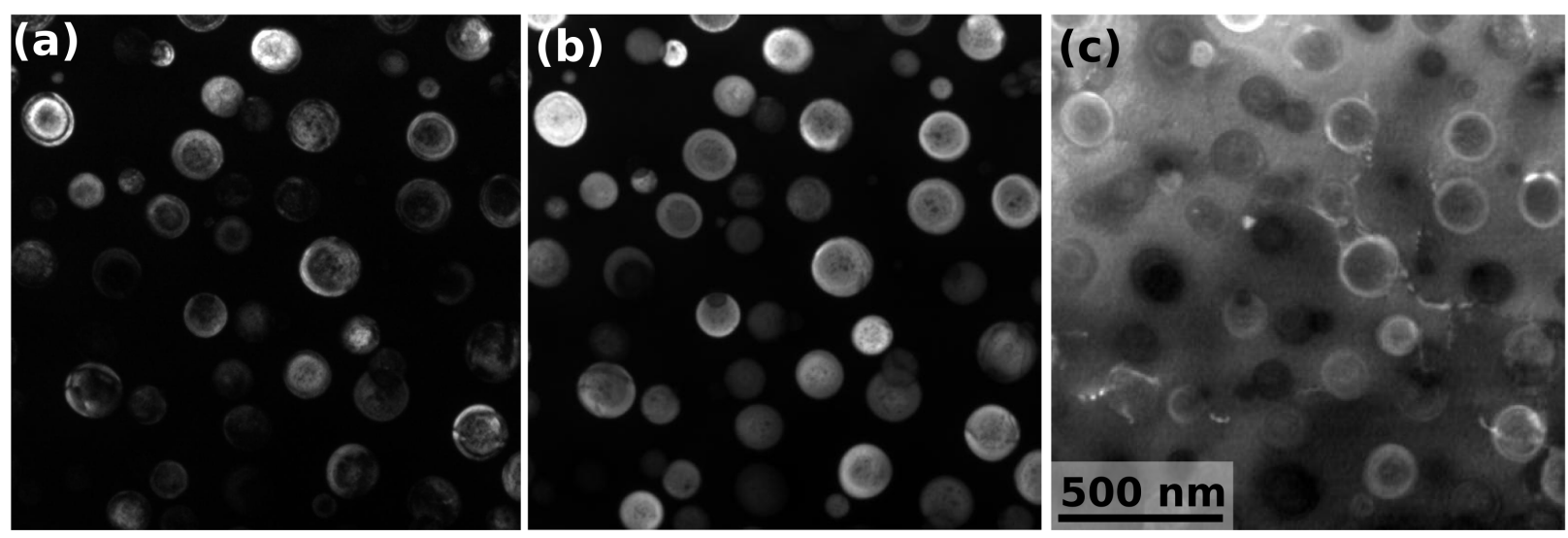

Figure 4: Comparison of individual with summed dark-field images: The image formed by summing dark-field images of the B2 sup7erlattice reflections (b) reveals the circular B2 precipitates better than an individual dark-field image (a).The conical dark-field image (c) shows a strong strain contrast revealing dislocation structures.

\subsection{STEM Diffraction Mapping}

Here we present an alternative way of mapping the combined real- and diffraction-space using STEM. Figure 5a shows an ADF-STEM image of the same sample position as illustrated in Figure 3. Figure $5 \mathrm{~b}$ shows the diffraction pattern of the matrix that was obtained by summing all diffraction patterns from the region indicated by the blue square in (a). The diffraction pattern shows the same result that was deduced in Figure 3a (bcc structure, [100] orientation). Figure $5 c$ is the diffraction pattern corresponding to the precipitate indicated by the red circle in (a), again establishing the B2 structure similar to Figure 3c. The diffraction pattern of the facetted precipitate indicated by the green triangle in (a) coincides with the one observed in Figure 3d, revealing a $\mathrm{Fe}_{23} \mathrm{Zr}_{6}$ precipitate. The present results show how diffraction patterns can be reconstructed from arbitrarily shaped regions that can be selected with pixel-precision. 

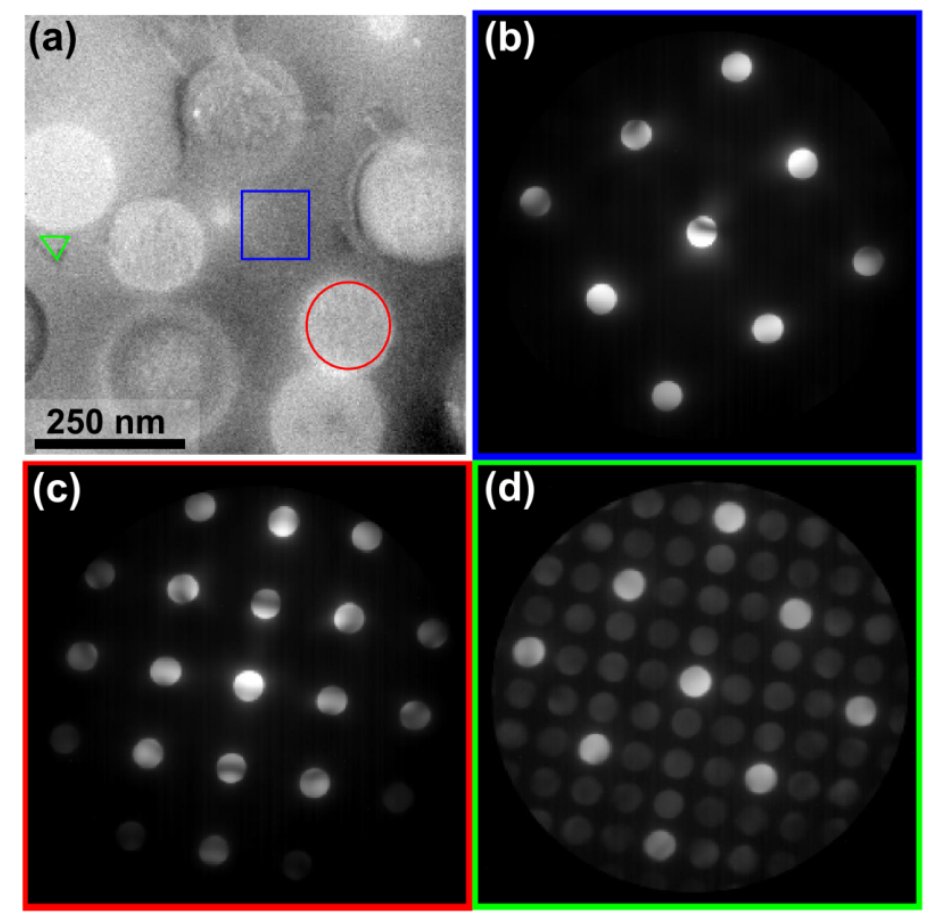

Figure 5: Virtual SA aperture: A STEM ADF image of the multiphase structure of Fe-Al-Ni-Cr alloy is shown in (a). A diffraction pattern was recorded for every probe position making it possible to use virtual selected area apertures for distinguishing the different phases. Figure (b) was calculated by summing the diffraction pattern in a matrix region (blue square). The diffraction pattern resulting from selecting a circular precipitate (red circle) is shown in (c). In addition a smaller precipitate was selected (greed region) leading to the diffraction pattern shown in (d).

The data-cube acquired by STEM diffraction-mapping can also be used to compute virtual dark-field images. Figure 6a illustrates the sum of all the diffraction patterns in the data-cube. The contrast was enhanced to visualize weak reflections. Representative virtual dark-field images displayed in Figure 6 demonstrate the versatile applicability of STEM diffraction-mapping to reconstruct various dark-field images from the same dataset of a complex microstructure. The virtual objective aperture indicated by blue circles in Figure 6a was designed to only select bcc-matrix reflections and the corresponding virtual dark-field image is given in Figure $6 \mathrm{~b}$. The main contrast in the image is due to scattering in the bcc-Fe matrix and residual contrast is observed from the B2-particles. Figure $6 \mathrm{c}$ was reconstructed using the B2 superlattice reflections only as indicated by red circles in Figure 5a and contrast is exclusively observed from the B2-NiAl precipitates. The Figure $6 \mathrm{~d}$ was reconstructed by using the superlattice reflections highlighted by the green circles in Figure 6a only belonging to the $\mathrm{Fe}_{23} \mathrm{Zr}_{6}$-type precipitate. Hence, the triangular precipitate lights up. In addition, speckle like contrast within some primary precipitates is observed. To confirm the structure of these small speckle-like precipitates, their STEM diffraction pattern was analyzed as well. It is interesting to note that the image contains some diffraction contrast leading to dark contrast in the vicinity of the projected dislocation line. In addition, bright contrast lines are observed at the matrix-precipitate interface of Figure $6 \mathrm{~d}$ similar to that obtained by ADF-STEM of Figure $2 \mathrm{~b}$. This 
suggests that elastic strain fields at coherent interfaces can also be imaged using virtual apertures. The present results clearly show how STEM diffraction-mapping can be used to image the different phases by using specifically designed virtual apertures that allow one to select multiple beams from the same datacube with high precision.
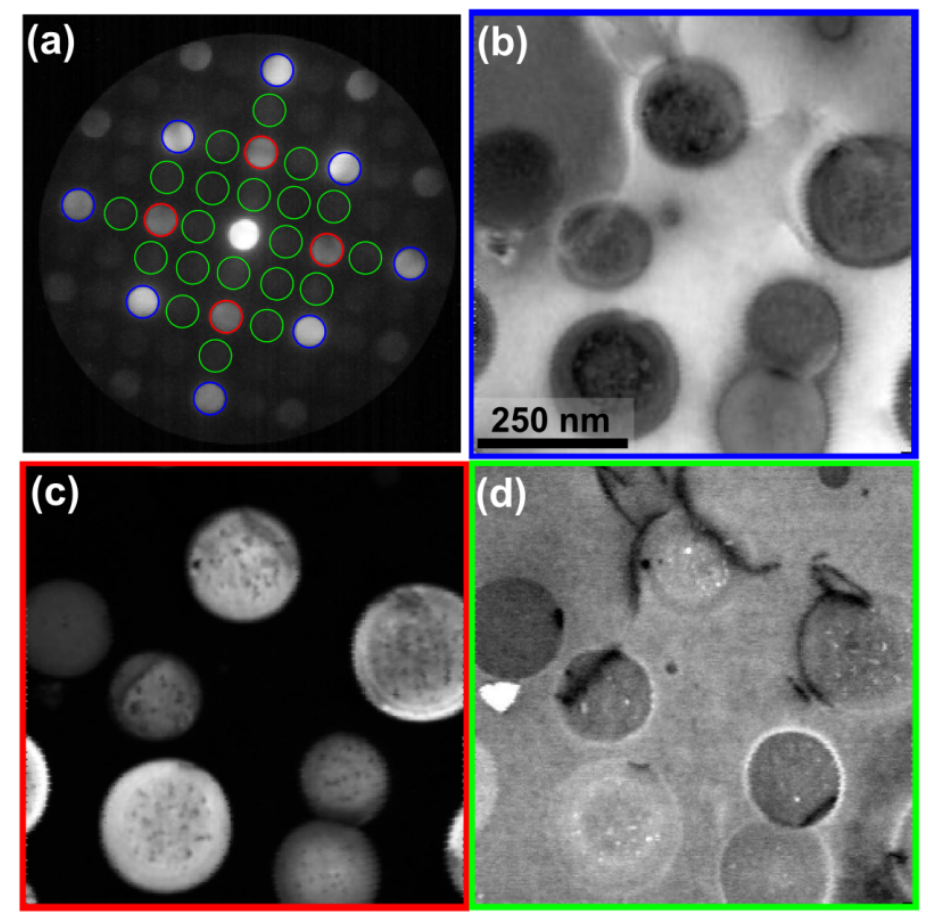

Figure 6: Virtual objective apertures: (a) Summed STEM diffraction pattern of the Fe-Al-Ni-Cr alloy showing reflections from different phases in the sample. Figure (b) shows an image that was reconstructed using the bcc reflections (the area used is indicated with the blue circles in (a)). The bccmatrix structure lights up. Figure(c) was calculated using the B2 superlattice reflections (indicated by red circles in (a)), the circular precipitates light up. For Figure (d) reflections belonging to the $\mathrm{Fe}_{23} \mathrm{Zr}_{6}$ structure but not the other two structures were used. In addition to the triangular precipitate speckles in the circular precipitate light up. 


\subsection{Comparison of Virtual BF/DF with BF/DF}
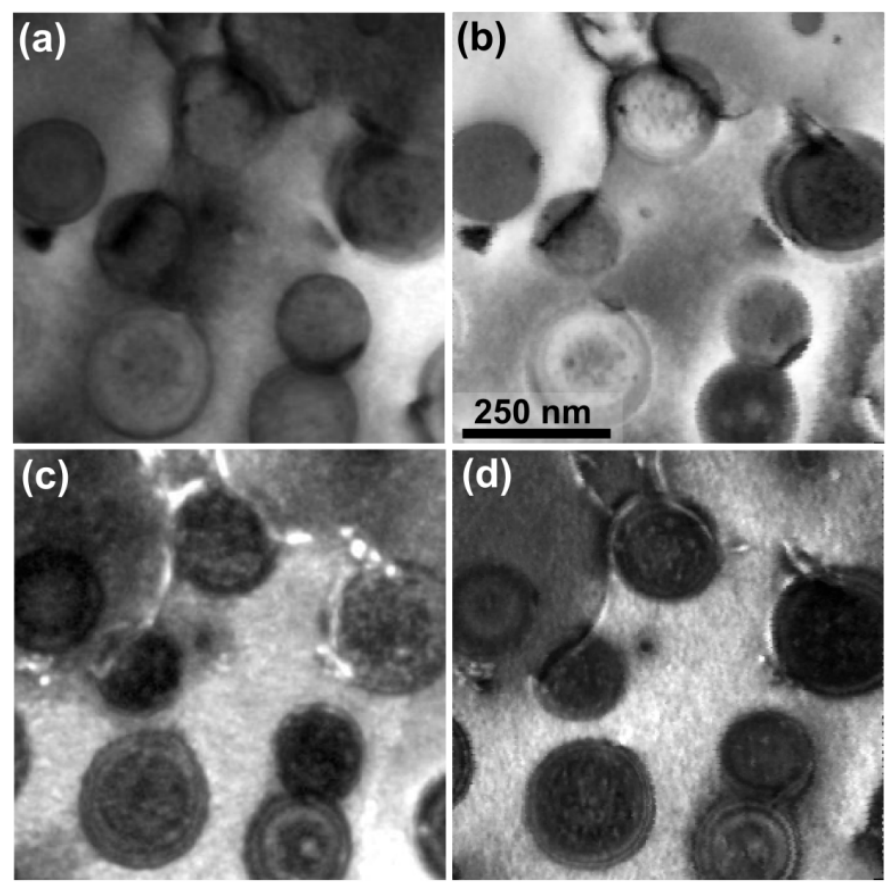

Figure 7: Comparison of STEM diffraction-mapping with conventional imaging: The conventional bright-field image (a) shows similar contrast when compared to the image reconstructed from the data-cube using a virtual objective aperture (b). The (100) dark-field image shown in (c) is comparable to the image formed by placing a virtual objective aperture on the same (100) reflection (d) but shows more dynamical contrast.

The applicability of STEM diffraction-mapping can easily be determined by comparing reconstructed virtual images with single TEM dark-field images. Figure 7a shows a conventional bright-field image formed using an objective aperture and acquired in zone-axis orientation. In Figure $7 \mathrm{~b}$ the bright-field image was reconstructed from the data-cube acquired by STEM diffraction-mapping by placing a virtual objective aperture around the transmitted beam (a diameter of $0.7 \mathrm{~nm}^{-1}$ was used corresponding to a collection angle of $1.4 \mathrm{mrad}$ ). A qualitative comparison reveals similar contrast in both images. Diffraction contrast is the dominating mechanism in both images seen in the strong negative contrast along projected dislocation lines. The reconstructed image seems to be less affected by effects like bending and dynamical scattering as the image results from the incoherent sum of images formed by different beam directions. Furthermore the signal to noise ratio is very good, as the exposure time of the STEM diffraction map is significantly larger than that of a single dark-field image (164 s as compared to $5 \mathrm{~s}$ ). It should be pointed out that in the present case a similar contrast can be achieved by using TEM diffraction mapping and summing over multiple images. , Figure $7 \mathrm{c}$ demonstrates a single dark-field image formed using a (100) reflection and Figure $7 \mathrm{~d}$ a reconstructed image formed by placing a virtual 
objective aperture around the same reflection. The contrast in both images is similar and both the precipitate and dislocation structure is resolved.

\section{Discussion}

The current paper describes two techniques in which relatively large datacubes are recorded and post-processing gives the microscopist much more flexibility and analytical potential than is possible by acquiring only an individual BF or DF image. Two approaches using large datacubes were compared: TEM and STEM diffraction mapping. In the case of TEM, the availability of all darkfield images not only allows one to improve the contrast by summing images selected by setting a virtual objective aperture but also allows one to reconstruct a diffraction pattern from a region with an arbitrary shape or a size as small as a pixel. In STEM diffraction mapping the fact that a full diffraction pattern is associated with each pixel of the data cube allows for the reconstruction of dark-field images from any number of different phases existing in one dataset, which is impossible by CTEM. The comparison with conventional imaging shows that dynamical scattering effects can be reduced. In the case of STEM diffraction mapping this can be achieved by using a convergent probe and in the case of TEM diffraction mapping by using different beam incident directions while multiple different dark-field images can be summed. The advantage of diffraction mapping over conventional microscopy is that the virtual aperture can in principle take any shape or size and arbitrary regions can be selected to reconstruct virtual images. Thus, the image contrast can be optimized by specifically designing the virtual aperture. Furthermore, virtual apertures as small as an individual pixel can be set while in CTEM the selected area aperture cannot be made as small as possible due to errors arising from aberrations leading to a displacements between the selected area from which direct and diffracted beams come [20]. 


\begin{tabular}{|c|c|c|c|c|}
\hline \multirow[b]{2}{*}{ Free parameters: } & \multicolumn{2}{|c|}{ STEM diffraction mapping } & \multicolumn{2}{|c|}{ TEM diffraction mapping } \\
\hline & general case & present case & general case & present case \\
\hline Image size & Scan raster $n$ & 256 & Detector size $x$ & 1024pixel \\
\hline Field of view (FOV) & $n \cdot$ stepsize & $700 \mathrm{~nm}$ & & $1500 \mathrm{~nm}$ \\
\hline $\begin{array}{l}\text { Diffraction pattern image } \\
\text { size }\end{array}$ & Detector size $x$ & $\begin{array}{l}\text { 2000pixel ( } 250 \\
\text { binning 8) }\end{array}$ & Tilt raster $n$ & 50 \\
\hline Reciprocal space range & $q_{\max }$ & $8.5 \mathrm{~nm}^{-1}$ & Tilt range $q_{\max }$ & $12 \mathrm{~nm}^{-1}$ \\
\hline Exposure time & $t$ & $0.0025 \mathrm{~s}$ & $t$ & $5 \mathrm{~s}$ \\
\hline $\begin{array}{l}\text { STEM convergence / TEM } \\
\text { collection angle }\end{array}$ & $\alpha$ & $\begin{array}{l}0.6 \mathrm{~nm}^{-1} \\
(1.2 \mathrm{mrad})\end{array}$ & $\begin{array}{l}\text { Aperture } \\
\text { radius } r_{q}\end{array}$ & $0.65 \mathrm{~nm}^{-1}$ \\
\hline \multicolumn{5}{|l|}{ Derived parameters: } \\
\hline Spatial resolution & $r e s=0.61 / \alpha$ & $1 \mathrm{~nm}$ & $r e s=0.61 / r_{q}$ & $1 \mathrm{~nm}$ \\
\hline Real space sampling points & $n^{2}$ & $6.55 \cdot 10^{4}$ & $x^{2}$ & $1 \cdot 10^{6}$ \\
\hline $\begin{array}{l}\text { Reciprocal space sampling } \\
\text { points }\end{array}$ & $x^{2}$ & $\begin{array}{l}4 \cdot 10^{6}\left(6.25 \cdot 10^{4}\right. \\
\text { binning } 8)\end{array}$ & $\begin{array}{l}\pi / 4 n^{2} \text { (circular } \\
\text { cutoff) }\end{array}$ & 1961 \\
\hline Dataset size (at 2 bytes) & $2 \cdot n^{2} \cdot x^{2}$ & $\begin{array}{l}500 \mathrm{~GB}(8 \mathrm{~GB} \\
\text { binning } 8)\end{array}$ & $2 \cdot n^{2} \cdot x^{2}$ & $4 \mathrm{~GB}$ \\
\hline Total acquisition time & $T=t \cdot n^{2}$ & $164 \mathrm{~s}$ & $T=t \cdot x^{2}$ & $9800 \mathrm{~s}$ \\
\hline
\end{tabular}

Table 1: Comparison of STEM and TEM diffraction-mapping. Parameters that can be tuned by the microscopist and some important derived parameters are shown for both methods. For both methods a general formula is given together with the value for the experiments of the present paper.

Table 1 shows a comparison between relevant experimental parameters comparing TEM and STEM diffraction mapping. The real and reciprocal space image sizes are different for both methods, but can be adjusted in the experiment. For STEM diffraction mapping the real space image size is limited by the size of the scanning raster, for TEM diffraction mapping by the size and binning of the CCD camera. The reciprocal space image size in STEM is limited by the size and binning of the electron detector, in the TEM case by the tilt raster. In the present case the sampling points in reciprocal space are significantly higher using STEM with respect to TEM diffraction mapping. 
Comparing the real and reciprocal space resolution of both methods, it is obvious that for STEM diffraction mapping the real space resolution is limited by the electron energy, the spherical aberration coefficient and the half-convergence angle [21]. If a small convergence angle is used, yielding separated diffraction discs, the convergence angle is the limiting factor for the spatial resolution. Defect imaging however can still be performed very effectively in STEM if a larger convergence angle is used [7,8]. In TEM diffraction mapping the resolution is limited by the size of the chosen objective aperture, where for the present case the spatial resolution was equal for both methods. The resolution in reciprocal space for STEM diffraction mapping is mainly limited by the half-convergence angle of the electron probe, which can be adjusted in the experiment. The reciprocal space sampling is much higher than the size of the diffracted discs, enabling not only the use of complex virtual apertures but also further quantitative evaluation of the diffraction patterns. The reciprocal space resolution in the case of TEM diffraction mapping is limited by the size of the aperture and the tilt raster used to acquire the dark-field tilt series.

While both methods are similar, STEM diffraction mapping is more time efficient, as short exposure times are sufficient for capturing the diffraction patterns. In fact the exposure time is only limited by the speed of the CCD or direct electron detector, while in the case of TEM diffraction mapping long exposure times are necessary to record dark-field images having low intensities. Therefore the total acquisition time and total dose in the case of TEM diffraction mapping is significantly higher, about a factor of 60 in the present experiment. In STEM diffraction mapping the entire diffracted signal is captured on the detector, while in the case of TEM diffraction mapping a significant fraction of the electrons are blocked by the objective aperture and are not collected. The fraction of signal collected can be estimated by comparing the aperture size to the DP size, $\left(r_{q} / q_{\max }\right)^{2}$, yielding in the present case $0.25 \%$. In the case of STEM diffraction mapping the signal is very high compared to detector readout noise, but in TEM diffraction mapping the achievable dynamic range can be increased by using a range of different exposure times.

The total data size and acquisition time of the recorded diffraction maps imposes further limitations. In TEM mode the acquisition of dark-field images is rather slow with a total acquisition time of $2.7 \mathrm{~h}$ for 2500 images in the present experiment. The acquisition even of a large STEM diffraction map with $512 \times 512$ pixels is possible within minutes using a fast direct electron detector such as the Gatan K2-IS. If such a detector is not available, highly binned diffraction patterns can be acquired on a regular CCD. The STEM diffraction maps acquired using a direct electron detector can get very large with up to $1 \mathrm{~TB}$ per single experiment if the whole detector is read out and a corresponding data storage infrastructure is needed. While high-resolution diffraction patterns can be beneficial for further analysis, for image reconstruction highly binned diffraction patterns with a size of $128 \times 128$ pixels are sufficient and therefore the size of the dataset could be reduced by binning the diffraction pattern or selectively reading out the detector to reduce the readout time.

\section{Conclusions and Outlook}


While electron microscopy has advanced substantially with the advent of aberration correction microscopy in the last decade it still relies mainly on the recording of individual images. Here we demonstrated how recording an entire datacube and using virtual apertures is not only feasible but also of great benefit for materials analysis. Diffraction mapping can also be used in an interactive way to inspect reconstructed dark-field images and diffraction patterns that change on the fly while the virtual aperture is moved around. Inspecting the data cube in this way allows one to analyze the data in much more detail than that is accessible with CTEM. When compared to commercially available diffraction mapping packages [14], the scripts used in the present paper allow for a very flexible and extendable use of the concept of virtual apertures, including the use of arbitrarily shaped apertures and the use of apertures containing arbitrary weights. Our approach is applicable to arbitrarily large datasets, as the scripts allow to process images one by one or load a downsampled datacube into memory for live processing.

In light of the recent interest in analysis of large data sets in many fields of science, we envision that the concept of virtual microscopy will open new research avenues in the field of electron microscopy. Novel ultrafast detector development paired with live computing will allow collecting multiple signals in parallel. The improvements of EDS detectors potentially makes it possible to combine STEM diffraction mapping with EDS, recording a spectrum at every pixel position [22]. Working with the entire datacube rather than with an individual image, not only allows for a detailed analysis of the structure but enables greater quantification of the microstructure. The diffraction patterns generated by TEM diffraction mapping or those acquired directly by STEM diffraction mapping can be used for phase or orientation mapping [23,24]. Recording diffraction patterns at high resolution also has the potential to generate strain maps by measuring the distance between diffracted peaks [25]. While in the field of X-ray diffraction the vast information contained in diffraction patterns is already widely exploited [26] similar techniques can be now be applied with electron microscopy but at sub-nm resolution. As demonstrated here, it is now possible to perform an electron microscopy experiment that generates a single large dataset containing all of the structural information in a sample and analyze it with virtual apertures to generate more quantitative and informative images than possible with real apertures. 


\section{Acknowledgments}

The authors thank Prof. Dunand (Northwestern University) for providing the alloy samples. We also acknowledge support by the Austrian Science Fund (FWF):[J3397] and the Molecular Foundry, Lawrence Berkeley National Laboratory, which is supported by the U.S. Dept. of Energy under Contract \# DE-AC02-05CH11231. C.H. Liebscher is thankful for the support by the German Research Foundation (Ll 2133/2-1).

\section{References}

[1] Hirsch PB, Howie A, Whelan MJ. Philos Trans R Soc A 1960;252:499.

[2] Bell W, Thomas G. Phys status solidi 1965;12:843.

[3] Yamaguchi S, Watanabe D, Ogawa S. J Phys Soc Jpn 1962;17:1030.

[4] Haider M, Epstein A, Jarron P, Boulin C. Ultramicroscopy 1994;54:41.

[5] Shibata N, Kohno Y. J Electron Micros 2010;59:473.

[6] Jesson D, Pennycook S. Proc Roy Soc Lond A 1995;449:273.

[7] Phillips P, Brandes M, Mills M, Graef M De. Ultramicroscopy 2011;111:1483.

[8] Agudo Jácome L, Eggeler G, Dlouhý A, Jácome LA, Eggeler G, Dlouhý A. Ultramicroscopy 2012;122:48.

[9] Perovic D, Rossouw C, Howie A. Ultramicroscopy 1993;52:353.

[10] Alloyeau D, Ricolleau C, Oikawa T, Langlois C, Le Bouar Y, Loiseau A. Ultramicroscopy 2008;108:656.

[11] Hirata A, Guan P, Fujita T, Hirotsu Y, Inoue A, Yavari AR, Sakurai T, Chen M. Nat Mater 2011;10:28.

[12] Rodenburg J, McCallum B, Nellist P. Ultramicroscopy 1993;48:304.

[13] Cowley J. Microsc Microanal 2004;10:9.

[14] Schaffer B, Gspan C, Grogger W, Kothleitner G, Hofer F. Microsc Microanal 2008;14 Suppl $2: 70$.

[15] Vo N, Liebscher C, Rawlings M, Mark Asta, Dunanda DC. Acta Mater 2014;71:89.

[16] Koch C, Özdöl V, Ishizuka K. Microsc Anal 2012;May:5. 
[17] Koch C. Ultramicroscopy 2011;111:828.

[18] Dingley DJ. Microchim Acta 2006;155:19.

[19] Abraham DP, Richardson JW, McDeavitt SM. Scr Mater 1997;37:239.

[20] Cockayne DJH, Chen Y, Li G, Borisenko KB. J Phys Conf Ser 2010;241:012006.

[21] Williams DB, Carter CB. Transmission Electron Microscopy A Textbook for Materials Science, vol. 2. Springer; 2009.

[22] Schlossmacher P, Klenov D, Freitag B, Harrach h. s. von. Micros Today 2010;18:14.

[23] Zaefferer S. Cryst Res Technol 2011;46:607.

[24] Liu H, Schmidt S, Poulsen H, Godfrey A. Science (80- ) 2011;332:833.

[25] Müller K, Rosenauer A, Schowalter M, Zweck J, Fritz R, Volz K. Microsc Microanal 2012;18:995.

[26] Hofmann F, Song X, Jun T, Abbey B. Mater Lett 2010;64:1302. 